\title{
Intervención educacional en profilaxis antibiótica para hernioplastias inguinales
}

\section{Educational intervention in antibiotic prophylaxis for groin hernioplasties}

\author{
Adolfo Galicia Armas, ${ }^{*}$ Ana Prixila González Vargas, * Carlos Eduardo Rodríguez Rodríguez, * \\ Alberto Robles Méndez Hernández, * Adriana Paz Mendoza*
}

\section{Resumen}

Introducción: Las hernias inguinales constituyen $75 \%$ de las hernias de la pared abdominal. Para su corrección, el tratamiento de elección es la hernioplastia con malla; un procedimiento limpio, cuya incidencia de infección del sitio quirúrgico es de 1 al $10 \%$. La profilaxis antibiótica, además, reduce este riesgo y los efectos negativos resultantes. Hoy en día, existe un desapego considerable a los protocolos de profilaxis antibiótica, pero las intervenciones educacionales pueden mejorar el apego a las guías de práctica clínica. Material y métodos: Se realizó un estudio prospectivo, comparativo, cuasiexperimental y no aleatorizado en 50 pacientes sometidos a hernioplastia inguinal con malla, quienes fueron divididos en dos grupos: $A(25)$ como control y B (25) como intervención. Se evaluó el impacto de una intervención educacional en cirujanos generales sobre la correcta administración de profilaxis antibiótica. Resultados: Hubo una selección correcta de antibiótico en cuatro pacientes del grupo A (16\%) en comparación con cuatro pacientes del B (16\%); administración antibiótica preoperatoria en 10 pacientes (40\%) del grupo A y en ocho pacientes (32\%) del grupo B ( $p=$ 0.55 ); duración del tratamiento no mayor a 24 horas en el grupo A de $10(40 \%)$ pacientes y en el grupo B de ocho pacientes $(32 \%)(p=0.11)$. Conclusiones: La intervención educacional en forma de folletos no generó impacto positivo en la correcta administración de la profilaxis antibiótica, sin embargo; sí generó tendencia a una mayor aplicación de profilaxis.

Palabras clave: Hernioplastia, profilaxis antibiótica, intervención educacional, pared abdominal.

\section{Abstract}

Introduction: Groin hernias encompass $75 \%$ of wall abdomen hernias. Gold standard treatment is hernioplasty with mesh, which consists in a clean wound procedure that could attend a surgical wound infection in 1 to $10 \%$ of all. Antibiotic prophylaxis reduces this risk and negative outcomes. There is detachment to antibiotic prophylaxis protocols, but educational interventions may improve the attachment to practice guidelines. Material and methods: A non-randomized, comparative, prospective, quasi-experimental research was carried out to 50 patients that underwent to hernia repair. They were distributed in 25 patients control group $A$ and 25 patients post intervention group B. Attachment to practice guidelines was assessed according to the appropriate prophylaxis conduct. Results: A 16\% draw (4 patients) between groups A \& B was observed related to correct antibiotic election. Group A showed 10 patients (40\%) and group B 8 patients (32\%) related to correct antibiotic election $(p=0.55)$. A greater number for treatment term less than 24 hours by group A was observed with 10 patients (10\%) vs group B with 8 patients $(32 \%)(p=0.11)$. Conclusion: Educational intervention through printed brochures were not enough to declare a positive impact on appropriate prophylaxis conduct, but an increase on antibiotic prophylaxis tendency was observed.

Keywords: Hernioplasty, antibiotic prophylaxis, educational intervention, abdominal wall.

\section{INTRODUCCIÓN}

* Facultad Mexicana de Medicina de la Universidad La Salle, México.
Servicio de Cirugía General, Hospital Ángeles Metropolitano. Ciudad
de México. Correspondencia:

Dra. Ana Prixila González

Correo electrónico: vargaddra.prixila@hotmail.com

Aceptado: 16-10-2019.

www.medigraphic.com/actamedica

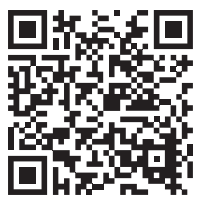

El $75 \%$ de las hernias de la pared abdominal está localizado en la región inguinofemoral. Para tratar este tipo de hernias, la hernioplastia se ha posicionado como el método quirúrgico de elección. Dicho procedimiento es uno de los más realizados en cirugía general, con aproximadamente 20 millones de hernioplastias anuales en el mundo.

De acuerdo con algunos reportes, ${ }^{1-3}$ el uso de mallas en la reparación herniaria ha permitido reducir la recurrencia en hasta a $1 \%$ para hernias primarias y hasta 
5\% para hernias recidivantes. La malla colocada en la reparación del defecto herniario induce la infiltración celular inflamatoria aguda, la cual es reemplazada por fibroblastos y células gigantes con respuesta fibroblástica del organismo a los polímeros de la malla, lo que da como resultado el desarrollo de una gruesa capa fibrosa alrededor de la malla que cubre y fortalece la zona herniaria.

La hernioplastia inguinal con malla está catalogada como un procedimiento limpio; sin embargo, existen algunos factores que pueden favorecer la infección del sitio quirúrgico. Su incidencia ha sido reportada del 1 al $10 \%$, según de la serie consultada. ${ }^{4}$ Los principales factores de riesgo para la infección del sitio quirúrgico son el descontrol de la glicemia, la hipotermia en el periodo postoperatorio, la presencia de obesidad (IMC > 30), la medicación con esteroides o fármacos inmunosupresores, el inmunocompromiso, la transfusión sanguínea, si se ha realizado hernioplastia de urgencia, un tiempo quirúrgico $>180$ minutos, una enterotomía concomitante a la plastia, y tener edad avanzada y tabaquismo. ${ }^{2}$ El desarrollo de infección del sitio quirúrgico depende de la dosis de contaminación, la virulencia de los patógenos y la resistencia a la infección del paciente; no obstante, la dosis de microorganismos contaminantes requerida para producir una infección puede ser mucho más baja en presencia de materiales protésicos, como las mallas.

La profilaxis antibiótica previene la infección del sitio quirúrgico; en estudios comparativos, se ha observado una incidencia de infección de $9.4 \%$ en pacientes sin profilaxis y de $2.8 \%$ en pacientes que sí recibieron antibiótico. ${ }^{2,5}$ A pesar de que la profilaxis antibiótica puede reducir la incidencia de infecciones del sitio quirúrgico hasta cuatro veces, ésta se utiliza en mayor medida en procedimientos de urgencia respecto a procedimientos electivos (50 en comparación con $16 \%$, respectivamente), lo que incrementa la probabilidad de infecciones del sitio quirúrgico de estos últimos. ${ }^{5,6}$ Asimismo, la infección del sitio quirúrgico incrementa los costos, debido a la necesidad de una segunda cirugía, dehiscencia de la herida quirúrgica, pérdida de la función de la pared abdominal e incremento en los días de estancia hospitalaria; además, genera un impacto negativo en la percepción de satisfacción del paciente, por lo que la profilaxis antibiótica es una medida crítica necesaria para reducir todos estos efectos negativos. ${ }^{2,4,6,7}$

En México, las Guías de práctica clínica para la prevención y diagnóstico de la infección del sitio quirúrgico recomiendan aplicar profilaxis antibiótica en hernioplastias con cefalotina a dosis de $1 \mathrm{~g}$ para pacientes con peso igual o menor a $80 \mathrm{~kg}$; de $2 \mathrm{~g}$ para pacientes con peso entre 80 y $120 \mathrm{~kg}$, y de $3 \mathrm{~g}$ para aquéllos con peso corporal superior a $120 \mathrm{~kg}$. En caso de haber alergia a betalactá- mico, es necesario utilizar clindamicina o vancomicina. También, estas guías sugieren aplicar el antibiótico no más de tres horas previas al procedimiento y durante un mínimo de 30 minutos antes de incidir la piel, aunque idealmente deberían administrarse entre 30 y 60 minutos antes de la incisión, ya que, de lo contrario, se reduce la efectividad del antibiótico e incrementa el riesgo de infección del sitio quirúrgico. Finalmente, mencionan que una sola dosis de antimicrobiano puede ser suficiente, pero extender la profilaxis a 24 horas puede reducir el riesgo de infección. ${ }^{5,8}$

La American Society of Health-System Pharmacists (ASHP) también recomienda que la profilaxis debe administrarse como dosis única o tener una duración no mayor a 24 horas. ${ }^{9}$ De igual manera, la SIGN (Scottish Intercollegiate Guidelines Network) sugiere que la profilaxis antibiótica debe administrarse entre 30 y 60 minutos previos a la incisión quirúrgica, pero si el procedimiento quirúrgico excede dos semividas del antimicrobiano utilizado o existe un sangrado significativo (>1,500 mL), entonces habrá que agregar una dosis adicional transoperatoria para asegurar concentraciones del fármaco suficientes en suero y tejido. Esta redosificación debe medirse a partir de la administración de la dosis preoperatoria y no desde el inicio del procedimiento. ${ }^{5,6,8}$

A pesar de todas estas recomendaciones, existe un desapego considerable a los protocolos de profilaxis antibiótica ya establecidos; las principales causas son el desconocimiento de la evidencia científica en la que se basan las guías clínicas, poca familiaridad y afinidad con las guías, y la inercia de la práctica previa (la cual suele condicionar renuencia al cambio). Una herramienta útil para mejorar el apego a las guías de profilaxis en cirugía es mediante las intervenciones educacionales. Se ha observado que después de una intervención educacional, puede haber un incremento hasta de $73 \%$ en el seguimiento de los protocolos correctos para aquellos procedimientos evaluados. ${ }^{6}$

En nuestro centro hospitalario, durante el periodo de enero de 2011 a junio de 2016, se llevaron a cabo 1,411 hernioplastias de pared abdominal, es decir, aproximadamente 260 procedimientos por año, por lo que mejorar la práctica de la profilaxis antibiótica con apego a las guías establecidas para estos procedimientos, puede generar un impacto positivo importante en la prevención de infecciones del sitio quirúrgico, disminuyendo costos, mejorando el grado de satisfacción del paciente y disminución de resistencias bacterianas. Por esta razón, es importante aplicar intervenciones educacionales de acuerdo con evidencia científica, para que se instruya a los cirujanos qué recomendaciones son establecidas en las guías de práctica clínica mexicanas. 


\section{MATERIAL Y MÉTODOS}

Se realizó un estudio prospectivo, comparativo, cuasiexperimental y no aleatorizado en el Hospital Ángeles Metropolitano de la Ciudad de México, en el que se evaluó el impacto de una intervención educacional sobre la correcta administración de profilaxis antibiótica en pacientes sometidos a hernioplastias inguinales con colocación de malla. Para ello, se tomaron como referencia las recomendaciones emitidas en las Guías de práctica clínica para la prevención y diagnóstico de la infección del sitio quirúrgico.

Se incluyeron en total 50 expedientes de pacientes mayores de 18 años, quienes fueron sometidos a hernioplastia inguinal con malla. Este total se dividió en dos grupos: A y B. El grupo A lo conformaron 25 pacientes sin intervención educacional de su cirujano, en tanto que el grupo B fueron 25 pacientes con intervención educacional del cirujano. Se registró la información acerca del tipo de antibiótico aplicado, el momento en que se aplicó la primera dosis de antibiótico y la duración del tratamiento. Se consideró correcta la administración de cefalotina y/o cefalosporina de primera generación, la aplicación de la primera dosis de antibiótico entre los 30 y 60 minutos antes de la incisión quirúrgica de la piel, así como la aplicación en la sala de preparación quirúrgica, y finalmente la duración del tratamiento no mayor a 24 horas. Se excluyeron expedientes de pacientes con alergia a betalactámico, comorbilidades, tabaquismo, algún tipo de inmunosupresión y expedientes con información incompleta.

Inicialmente, se revisaron 25 expedientes procedentes del archivo clínico hospitalario (grupo A), tomados de manera consecutiva a partir del primero de julio de 2012 y se registró la información en una base de datos. Posteriormente, en la sala preoperatoria y en el momento preoperatorio, se les entregó por escrito y explicó verbalmente a los cirujanos programados para realizar hernioplastia inguinal con malla las recomendaciones de profilaxis antibiótica para hernioplastias de acuerdo con las Guías de práctica clínica para la prevención y diagnóstico de la infección del sitio quirúrgico del Instituto Mexicano del Seguro Social. Los cirujanos fueron seleccionados de manera consecutiva a partir del primero de mayo de 2018 y los expedientes de los pacientes de dichos cirujanos (grupo B) fueron revisados posterior al alta hospitalaria para la recopilación de datos. Finalmente, se compararon y analizaron los datos obtenidos de ambos grupos para determinar si hubo alguna diferencia en la profilaxis antibiótica administrada en pacientes operados por cirujanos sin intervención educacional, respecto a la profilaxis antibiótica en aquellos operados por cirujanos que sí recibieron intervención educacional.

Para el análisis estadístico se utilizó el Statistical Package for Social Sciences, versión 21 (SPSS Inc., Chicago, IL,
USA), así como Microsoft ${ }^{\circledR}$ Office Excel, versión 14.3.0. Se aplicó prueba de Levene para la igualdad de varianzas; para variables cuantitativas de razón, se calculó la media como medida de tendencia central, y desviación estándar (DE) como medida de dispersión; para variables cualitativas nominales, se calculó proporción y prevalencia como medidas de frecuencia, con prueba de $\chi^{2}$ para comparación de muestras independientes con valor significativo de $p \leq 0.05$.

\section{RESULTADOS}

Se evaluó la profilaxis antibiótica administrada a 50 pacientes divididos en dos grupos: en el grupo $A$, sin intervención, y en el grupo B, con intervención. En cuanto a los datos demográficos, el grupo A se conformó por seis mujeres (24\%) y 19 hombres $(76 \%)$, con un promedio de edad de 46.7 años y una DE 11.1 ; el grupo B se formó con ocho mujeres (32\%) y 17 hombres (68\%), con edad promedio de 49.1 años y DE 15.0; el análisis de varianzas, la edad tuvo una $p=0.78$, y el sexo una $p=0.52$, sin diferencia estadística entre grupos para edad y sexo.

En cuanto a la selección del antibiótico correcto, las guías recomiendan cefalotina. En ambos grupos se obtuvo el mismo resultado: cuatro pacientes (16\%) de cada grupo indicaron el antibiótico correcto, sin observar ninguna diferencia al respecto. Cabe señalar que en ambos grupos el antibiótico que más frecuentemente se administró fue ceftriaxona con 10 pacientes (40\%) en el grupo A y 18 (72\%) pacientes en el grupo B. Se observó también que, después de la intervención educacional, hubo mayor administración de profilaxis: con 20 pacientes (80\%) en el grupo A comparación con 24 pacientes (96\%) en el grupo B, aunque esto no tuvo impacto en la correcta selección del antibiótico.

Respecto al momento de la administración, las guías recomiendan que sea antes de la incisión quirúrgica (en el periodo preoperatorio) e idealmente entre el minuto 30 a 60 antes de la incisión. En nuestro estudio, se observó la aplicación del antibiótico en periodo preoperatorio en 10 pacientes del grupo A (40\%) y en ocho pacientes del grupo B (32\%); $p=0.55$. En el periodo transquirúrgico, se aplicó a 11 pacientes del grupo A y 13 pacientes del grupo B, y en el periodo postoperatorio se administró en cuatro pacientes para cada grupo.

La duración del tratamiento recomendada es de no más de 24 horas. En nuestros grupos, para el grupo A se suspendió el tratamiento a las 24 horas en 10 pacientes $(40 \%)$ y para el grupo B en ocho pacientes (32\%), con $\mathrm{p}=0.11$ (Tabla 1). 
Tabla 1:

\begin{tabular}{|c|c|c|c|c|c|}
\hline & \multicolumn{2}{|c|}{ Grupo A, n (\%) } & \multicolumn{2}{|c|}{ Grupo B, n (\%) } & \multirow[b]{2}{*}{$p$} \\
\hline & Correcto & Incorrecto & Correcto & Incorrecto & \\
\hline Selección de antibiótico & $4(16)$ & $21(84)$ & $4(16)$ & $21(84)$ & NS \\
\hline $\begin{array}{l}\text { 1a. dosis en sala preoperatoria (30-60 } \\
\text { min antes de la incisión quirúrgica) }\end{array}$ & $10(40)$ & $15(60)$ & $8(32)$ & $17(68)$ & 0.55 \\
\hline $\begin{array}{l}\text { Duración de tratamiento antibiótico } \leq \\
24 \text { horas }\end{array}$ & $10(40)$ & $15(60)$ & $8(32)$ & $17(68)$ & 0.11 \\
\hline
\end{tabular}

$p=$ valor significativo $\leq 0.05 ; \mathrm{NS}=$ no significativo.

\section{DISCUSIÓN}

González VAP y colaboradores evaluaron la profilaxis antibiótica en 71 pacientes sometidos a distintos tipos de hernioplastias de pared abdominal anterior con y sin maIla, en el mismo centro hospitalario donde se realizó este estudio. Ellos encontraron que a $77 \%$ de los pacientes se les aplicó profilaxis antibiótica, un porcentaje muy similar al obtenido en nuestra serie para el grupo sin intervención (80\%). No obstante, esperaríamos que este porcentaje fuera mucho mayor en nuestro estudio por incluir solamente hernioplastias inguinales con colocación de material protésico. En cuanto a la correcta selección del antibiótico, ésta fue de 21.8 contra $16 \%$ en nuestra serie. Finalmente, en cuanto al momento de aplicación de la primera dosis de antibiótico en el periodo preoperatorio, hubo mayor diferencia, siendo de 21 contra $40 \%$ a favor de nuestro estudio. Si comparamos con los nuestros los resultados obtenidos del estudio de González en el grupo en los cirujanos que sí recibieron la intervención educacional, podemos ver incremento en la aplicación de profilaxis en 77 contra $96 \%$ en nuestro estudio, y con diferencia en cuanto a la selección del antibiótico con 21.8 en comparación con $16 \%$ a favor del estudio de González y otros. ${ }^{9}$

Por su parte, Newland revisó 8,765 casos en donde se utilizaron antibióticos de amplio espectro. Como resultados, encontró que los antibióticos más comunes fueron ceftriaxona/cefotaxima con $43 \%$, seguido de vancomicina con el $18 \%$, ceftazidima con $12 \%$ y meropenem con $7 \%$. En nuestro estudio, en el grupo A sin intervención, se utilizaron antibióticos de amplio espectro de manera incorrecta en $60 \%$ de los pacientes, y tras la intervención educacional, de modo contradictorio, aumentó hasta $80 \%$, siendo predominante el uso de ceftriaxona en ambos grupos con 40 y $72 \%$, respectivamente; estas cifras fueron similares a lo reportado por Newland. ${ }^{10}$
Al evaluar el impacto de la intervención educacional que hubo en nuestro estudio, observamos que aunque hubo un incremento en el porcentaje de aplicación de la profilaxis (80 contra 96\%), la selección del antibiótico empleado fue errónea en la mayoría de los casos (86\%) para ambos grupos. Meeker, en un ensayo clínico aleatorizado realizado en 2016, evaluó la prescripción inadecuada de antibióticos en pacientes con infecciones inespecíficas del tracto respiratorio alto y aplicó de una a tres intervenciones educacionales de las prescripción de medicamentos a las que llamó: "estrategia de alternativas sugeridas", en donde se hacían recomendaciones de tratamiento sin antibióticos; "estrategia de justificación responsable" que requerían justificar por escrito la prescripción seleccionada, y "estrategia de comparación" en la que debían comparar su prescripción con la realizada por otros colegas con una prescripción más acertada de acuerdo con las guías de práctica clínica. Con estas tres intervenciones, encontró una disminución de 23.2 a $5.2 \%$ para la estrategia de justificación responsable ( $p<0.001)$; y de 19.9 a 3.7\% para la estrategia comparación entre pares $(p<0.001)$; sin encontrar interacciones estadísticamente significativas de sinergia ni de interferencia entre las intervenciones. Probablemente, sus resultados hayan tenido un mayor impacto positivo por haber realizado hasta tres intervenciones en algunos casos, y por tratarse de intervenciones que, además de informar, estaban diseñadas para que el médico analizara su prescripción, mientras que nosotros realizamos una sola intervención y de carácter informativo. ${ }^{11}$

Goff también demostró que las intervenciones educacionales sistematizadas y etapificadas con el objetivo de cambiar positivamente la vía de administración, la dosificación y la sustitución de medicamentos pueden contribuir al éxito de los programas de calidad en la administración de antibióticos y generar ahorros de hasta 832,590 dólares anuales para el gobierno; también señala que la educación 
clínica puede mejorar significativamente la prescripción antibiótica, pero que existen factores complejos que afectan el comportamiento humano, como creencias y costumbres, las cuales generan resistencia al cambio. Por ello, menciona que se requiere colaborar con ciencias sociales y ciencias del comportamiento para el desarrollo de programas de intervención educacional, requiriendo repetición rutinaria de dichos programas para lograr la modificación de la conducta. ${ }^{12}$

Lee realizó un metaanálisis acerca de los resultados derivados de la aplicación de distintos métodos de intervención educativa. Él encontró reducciones en la prescripción errónea de antibióticos de 25\% mediante seminarios interactivos, de $9 \%$ con campañas por correo, de $52 \%$ por medio de trabajo con grupos educacionales pequeños, de $30 \%$ con visitas educacionales y de $42 \%$ mediante guías clínicas y folletos. Este investigador señaló que es difícil comparar la efectividad entre los docentes y las distintas estrategias para poder definir qué intervención genera un mejor resultado; sin embargo, la intervención con educación en grupos pequeños parece ser la más efectiva. En nuestro estudio, a pesar del uso de folletos como principal herramienta didáctica e informativa, no obtuvimos resultados favorables como los reportados por Lee. ${ }^{13}$ Consideramos que, metodológicamente, el tamaño de la muestra utilizada pudo influir para no obtener resultados favorables, y que en nuestro centro hospitalario, la inercia de la práctica previa y el desconocimiento de la evidencia científica que sustentan las recomendaciones otorgadas son factores determinantes en el desapego a las recomendaciones asentadas en las guías de práctica clínica.

\section{CONCLUSIÓN}

El otorgamiento en el periodo preoperatorio de folletos con las recomendaciones de profilaxis antibiótica en hernioplastias, especificadas en las Guías de práctica clínica para la prevención y diagnóstico de la infección del sitio quirúrgico a los cirujanos generales, no generó algún impacto positivo en la correcta administración de la profilaxis antibiótica, sino que sólo se observó una tendencia a una mayor aplicación de profilaxis antibiótica, sin diferencia en cuanto a la correcta selección del antibiótico, el momento de administración de la primera dosis ni en la duración del tratamiento.
Los resultados obtenidos sugieren la realización de nuevos estudios que se enfoquen en determinar las causas de desapego a las guías de práctica clínica, y que a su vez, éstas puedan ser implementadas junto con intervenciones educacionales dirigidas a modificar las causas de desapego identificadas a través de técnicas didácticas más efectivas.

\section{REFERENCIAS}

1. Mehrabi M, Jabbari A, Jabbari M, Jangjoo A. The role of prophylactic cefazolin in the prevention of infection after various types of abdominal wall hernia repair with mesh. Asian J Surg. 2015; 38 (3): 139-144.

2. Zhuo Y, Zhang Q, Tang D, Cai D. The effectiveness of I.V. cefuroxime prophylaxis of surgical site infection after elective inguinal hernia repair with mesh: A retrospective observational study. Eur J Clin Pharmacol. 2016; 72 (9): 1033-1039.

3. Asociación Mexicana de Cirugía General. Tratado de cirugía general. 3a. ed. Ciudad de México: Manual Moderno; 2017. Capítulo 183, Hernia inguinal; p. 1517.

4. Bueno J, Torregrosa A, Sala A, Carbonell F, García P, Bonafé S et al. Predictors of mesh infection and explanation after abdominal wall hernia repair. Am J Surg. 2016; 213 (1): 50-57.

5. Asensio A. Infección de la localización quirúrgica. Profilaxis antimicrobiana en cirugía. Enferm Infecc Microbiol Clin. 2013; 32 (1): 48-53.

6. Cameron M, Jones S, Adedeji O. Antibiotic prophylaxis audit and questionnaire study: traffic light poster improves adherence to protocol in gastrointestinal surgery. Int J Surg. 2015; 19: 112-115.

7. Ross F, Jones N, Townend A, Bhaskar P. The cost of inappropriate antibiotic prophylaxis in inguinal hernia repair surgery. Int J Surg. 2015; 23: S72-S73.

8. Instituto Mexicano del Seguro Social. Prevención y diagnóstico de la infección de sitio quirúrgico. Guía de evidencias y recomendaciones: Guía de práctica clínica. México: IMSS; 2018. pp. 21, 27.

9. González VAP, Garza LH, Ponce PLV. Profilaxis antibiótica en hernioplastias de pared abdominal. Acta Med. 2015; 13 (4): 217-223.

10. Newland JG, Stach LM, De Lurgio SA, Hedican E, Yu D, Herigon JC et al. Impact of a prospective-audit-with-feedback antimicrobial stewardship program at a children's hospital. J Pediatric Infect Dis Soc. 2012; 1 (3): 179-186.

11. Meeker D, Linder JA, Fox CR, Friedberg MW, Persell SD, Goldstein $\mathrm{NJ}$ et al. Effect of behavioral interventions on inappropriate antibiotic prescribing among primary care practices: a randomized clinical trial. JAMA. 2016; 315 (6): 562-570.

12. Goff DA, Bauer KA, Reed E, Stevenson KB, Taylor JJ, West JE. Is the "low-hanging fruit" worth picking for antimicrobial stewardship programs? Clin Infect Dis. 2012; 55 (4): 587-592.

13. Lee CR, Lee JH, Kang LW, Jeong BC, Lee SH. Educational effectiveness, target, and content for prudent antibiotic use. Biomed Res Int. 2015; 2015: 214021. 